\title{
Sentiment Classification through Convolutional Neural Network Based Quick Sentiment Analysis
}

\author{
Kota Venkateswara Rao ${ }^{1,2}$, M. Shyamala Devi ${ }^{1}$ \\ ${ }^{1}$ Department of Computer Science and Engineering, Vel Tech Rangarajan Dr. Sagunthala R\&D Institute of \\ Science and Technology, Avadi, Chennai, TamilNadu, India. \\ ${ }^{2}$ Department of Computer Science and Engineering, Andhra Loyola Institute of Engineering and Technology, \\ Vijayawada, Andhra Pradesh, India
}

\section{ABSTRACT}

Sentiment Analysis (SA) is characterized as the region of study to analyze individuals' Sentiment, surveys, and mentalities from various languages or from web remarks. SA has spread to each conceivable space like budgetary, medicinal services, online items, internet business to get-togethers, media transmission, political causes and decisions. In the manuscript, a computerized framework, which forms a huge dataset of analysis for viewpoint based decisions is proposed. The sentiments are gathered by Natural Language Processing (NLP) and afterward it is assigned positive, impartial and negative. As the quantity of web information is exponentially expanding, it turns out to be increasingly imperative to create models to investigate the content information consequently. The text may contain different labels, for example, age, gender, nation, sentiment, review etc. Utilizing such labels may carry advantages to some modern fields, such huge numbers of investigations of classification of texts have showed up. As of late, the Convolutional Neural Network (CNN) has been utilized for the process of classification of text and has obtained better results. In the manuscript, Convolutional Neural Network based Quick Sentiment Analysis (CNN-QSA) is proposed for the assignment of sentiment classification. The most main reason for to utilize $\mathrm{CNN}$ in $\mathrm{SA}$ is that $\mathrm{CNN}$ can extract features from global data, and it can think about the relationship among these features. $\mathrm{CNN}$ has a convolutional layer to extract data by a larger part of text with convolutional neural system. The proposed CNN-QSA method is compared with the traditional methods and the results show that the proposed method is better than the traditional methods like SVM and Naive Bayes strategies.

Key words : Sentiment Analysis, Natural Language Processing, Textual Data, Text Classification, Convolutional Neural Network, Feature Extraction.

\section{INTRODUCTION}

Sentiment Analysis and Opinion mining is almost comparative one anyway there is slight assortment between them for example opinion mining separates and investigate individuals' conclusion about a substance while Sentiment analysis search for the slant words/articulation in a book and after that analyze it. Opinion mining is additionally represented as the mining that expects to choose the conclusion unrevealed about a content noted in regular language. This perceives and chooses the natural data from the quality materials [1]. Opinions uncovered in a gathering of source records regarding a text document are extricated with opinion mining. This is finished by picking the characteristics of an item from the client assertions and chooses whether the remarks are ideal or disappointing [2]. For instance, the items salespersons are focused more on each client singular remarks in web based with respect to the items and administrations. These client remarks have high significance in the assessments of different buyers. Establishments and business keep watch on these buyer remarks to know the method for items perceived [3] [4]. Although analyzing these things is one of the uncertain employments on the grounds that there are accessible sources that contain spacious information.

Assumptions indicate the sentiments; Sentiments are the thoughts of people for the particular item. Assumption investigation of sentiment mining is extremely a complex task. There are numerous confusions like characteristic language processing for automated extricating of features, ordering and editing Sentiments which are communicated in on the web. Opinion Analysis is an investigation that is practiced by different associations for recognizing client input about the items. This makes different clients for knowing the ideal determination of their supported item. Sentiment Analysis using machine learning methods is ordinarily utilized in opinion mining for knowing notions, subjectivities also delicate states in online reviews and opinions [5-8]. The procedure was practiced on product assessment by sorting out the items qualities. Right now, estimation and analysis of sentiments is used in a broad scope of areas like E-Commerce and health care applications. By using $\mathrm{CNN}$, the process of performing sentiment analysis is depicted in Figure 1. 


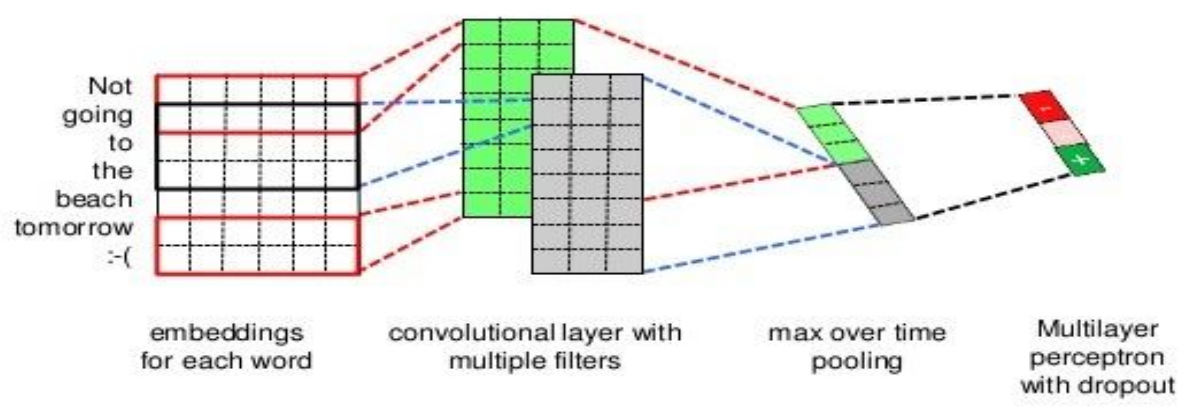

Figure 1: Process of Sentiment Analysis using CNN

In the web, numerous text information exists and it very well may be sorted out roughly into two most significant orders: truth and assumption. The fact of the matter is the target declaration though Sentiments are the emotional articulations. Sentiments are singular conclusions, emotions, assessments, and mentalities related with the particular item or product. The significant part of the sentiment analysis on text information has been engaged with respect to mining from truth based information, for example, online products, recovery of data, content gathering, content classification, and so on. Accessible numerous quantities of sentiment based sources and all may additionally have an enormous measure of Sentiment based informations using machine learning approach for attaining better results. Customer's expressions are generally occupied with broad discussion posts and sites for survey. It is badly arranged for the clients to blueprint such sources, finding right Sentiment articulations, understand them, and convert them into positive and negative assessments [9].

Sentiment Analysis focuses on the determination of user's perspective regarding explicit subject. The perspective includes appraisal, sensitivity or even passionate stages [10]. The most prime activity in opinion examination is the order of the extremity of explicit content at the degrees of features, archives, sentences and so forth. After text divisions are

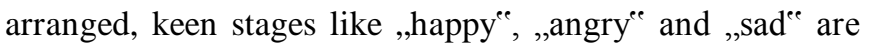
likewise recognized [11]. The arrangements of text divisions is the principle task in sentiment mining and it occurs at the hour of a segment of content expressing a Sentiment on a solitary issue that is classified as one among the two incompatible assessments. Hardly any models for extremity characterization are "like versus „dislike ${ }^{\text {ee }}$ or "thumbs upe versus ,thumbs down"e. This grouping likewise finds the favorable circumstances and damages of explanations in online surveys and helps with making the evaluation of items progressively solid. Another type of paired sentiment classification is understanding recognition of sentiments analyzed [12].

Sentiment Analysis is one of the unpredictable techniques that comprise of five significant stages for looking at inclined information. The sequence of Sentiment Analysis process is appeared in Figure 2. The stages incorporates, I. Gathering
Reviews, ii. Preparation of data, iii. Performing review analysis, iv. Sentiment classification, v. Generating Results.

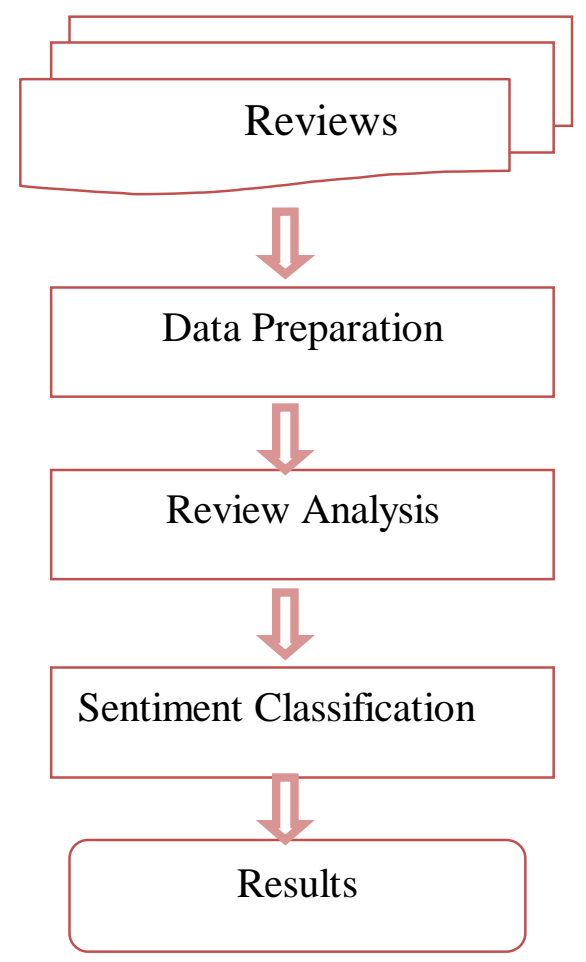

Figure 2: Sequence of Sentiment Analysis

The explanations that are chosen are examined and the explanations that are with a conclusion are protected though the other outstanding assertion. This undertaking can be conveyed in different levels or states, one term, whole sentence or entire record with commonly applied methodologies [13]. These procedures incorporate as follows:

$$
\begin{aligned}
& >\text { Unigrams } \\
& >\text { N-grams } \\
& >\text { Lemmas } \\
& >\text { Negation } \\
& >\text { Opinion words }
\end{aligned}
$$

Machine learning methodology is considered for getting accurate results in the process of sentiment analysis and for accurate classification of text based on sentiments identified and rating the products [14]. 


\section{LITERATURE SURVEY}

Y. Han et al. [1] introduced a independent feature extraction from the web information. This method portrays the KnowItAll framework. This framework is another structure thought, featuring its novel ability to infer the data without any hand-marked organized models. This framework focuses to computerize the standard procedure of determining the massive determination of realities, for instance, names of extraordinary pioneers or researchers from the web in an area free, solo and versatile way. So as to upgrade the frameworks review and inferring rate, there are three particular ways for assessing its exhibition; they are design learning, subclass extraction and rundown extraction. Sentiment model learning finds the explicit inferring decisions so that there are additional inductions that are likewise conceivable.

Z. Nasim et al. [2] handled parallel characterization utilizing the SVM and accomplished $71.6 \%$ exactness for Twitter data, where they embraced two sorts of target-autonomous features (e.g., twitter text highlights and supposition vocabulary features). Z. Xiangyu et al. [3] utilized the quantity of positive words and the quantity of negative words as features and accomplished $82.3 \%$ precision for double grouping with Twitter reviews. M. H. Krishna et al.[4] joined unigram features and their own Twitter-explicit features and acquired $80 \%$ precision for double characterization utilizing the SVM classifier. P. Yadav et al. [5] characterized the number supposition things as an element and joined the component with unigrams. They accomplished $75.2 \%$ precision for ternary arrangement utilizing the SVM.

D. V. N. Devi et al. [8] examined about finding assessments naturally on client conclusions. By exploring the assessments of the items from the clients, it is conceivable to separate the items which are missing and which are solid. This can be found with the assistance of sentiment examination. This makes to develop a model that can determine the Sentiment words consequently and find their extremity with the assistance of a lexicon. This limits the hand-worked exertion of dissecting these assessments and to assess them. It additionally depicts the significance of utilizing unstructured content in the spot of preparing information that are costly. A strategy is discovered that relies upon rules in which the item remarks are considered from reviews containing destinations and investigation is finished. Subsequently an individual should ready to know whether a particular item review is positive or negative or neutral.

The procedure for SA by means of R. Hegde et al. [9] depended on deep NLP of the sentences, using a part parsing as a advancement. Assessment Propagation is completely founded on the SA calculation that acknowledged that each semantics component is a kind of an issue. They showed a gathering of syntactic-based measurements that intended to cover a significant bit of the thought putting nature conveyed by content. They anticipated Associate in nursing information representation structure during which they expected to filter through numerous information questions or to contextualize the information with the objective that just the data essential to a customer request is given the impression to the customer. K. Han et al. [10] have shown a much customized framework for fine-grained Storm Troops on the sub sentence level, merging shifted supposition vocabularies and neighborhood and furthermore online review relations. They use Andrei Markov rule to facilitate furthest point scores from changed evaluation word references using data in regards to relations between neighboring segments. Their results incontestable that the utilization of fundamental features expanded the exactness of furthest point estimates achieving precision scores up to $69 \%$.

S. Jebbara et al. [11] use the phonetic examination to explore the idea in sentence level with the nearness of product review rates. This technique is introduced to infer the conclusions identified with the sentence or expression. Sentiment Analysis has been attempted for the most part for records regularly a Sentiment or another item. Conjunctions have a broad impact on the total sentiment of a sentence, along these lines, it discover a method for nuclear assessments of independent expressions incorporate with each other in the presence of conjuncts to decide the total sentiment of a sentence. The word conditions are used to inspect the sentence builds and along these lines, the outcome got about $80 \%$. It additionally analyzes the impact of WordNet on the yield precision.

Ghiassi et al. [12] contemplated that in numerous past works, item highlight and determining assessments are treated as two separate errands. Right now, are converged with each other by utilizing probabilistic models. This respects the trouble of item qualities and supposition extraction as a succession marking task and chooses the restrictive arbitrary field's models to accomplish this. A computational strategy is created to assemble area explicit assessment dictionary by incorporating semi-organized supposition with general notion vocabulary that helps with finding the notion directions of Sentiments. The adequacy of the proposed approach is appeared with the test results on two genuine world datasets.

Balazs et al. [13] studied about the conclusion mining and assumption investigation.

This overview portrays the strategies and methodology which guarantee to allow Sentiment focused data looking for frameworks straightforwardly. This focuses on these methodologies that mean to mark the new challenges raised by assumption mindful applications when contrasted with the one that exist already in a much broad truth based investigation. This includes the material on the portrayal of evaluative content and on more extensive issues concerning control, security and financial impact that the advancement of supposition arranged data procures administrations emerges. Chaturvedi et al. [14] proposed an assessment investigation utilizing Support Vector Machine. For Feature extraction, this proposed approach utilizes $\mathrm{N}$-grams, diverse weighting plans and furthermore investigates Chi-Square weight features. This Chi-Square weight gives a huge improvement in arrangement exactness. The assessment of the results in 
film related area shows conspicuous outcomes. AUC on unigram approach is 0.917 , though AUC on bi-gram approach is 0.728 . Text arrangement utilizes preparing and testing informational index from Pang Corpus, which has film surveys gathered from IMDb.

C.R.Bharathi et al. [15] proposed a multilayer perception model for Sentiment analysis and selection tree-based component positioning is proposed for feature determination. The author likewise proposed a crossover calculation, in view of differential development and genetic calculation for weight streamlining calculation to improve multilayer perceptron neural system is proposed. The technique is assessed on IMDb dataset. A multilayer perceptron is a feed-forward neural system; it can have at least one concealed layers alongside information and resultant layers. A multilayer perceptron has numerous node layers, where every node is neuron with non-direct enactment work in layers other than the information layer[16-22].

\section{DISADVANTAGES OF EXISTING SYSTEM}

With the advancement of internet based life, reviews on the web and its conversations, sentiments, proposals, gathering, criticisms and postings are utilizing in the media to make goals by people and associations. In like manner the total foundation extremity or client assumptions in regards to not many traits are sorted out utilizing Sentiment Analysis. The order is found as a significant test in Sentiment Analysis and the review can comprehend the item, assessment of item or evaluation of an item that can be a choice, record or quality which is gathered into positive or negative. Due to the communication of various locales in the web, it is difficult to watch and decide the destinations of client perspectives and determining the data. In the spots of long online journals and gatherings, all locales incorporate not many sentiment sentences and it very well may be comprehend with no troublesome.

The destinations relating the specific thing was found and determining, inspecting the perspectives is urgent through utilizing a normal human peruser. In these conditions, the arrangement of SA is essential. Right now, opinion analysis is the mandate at the convergence of NLP and recovery of data and furthermore parts the numeral of features other than with different procedures that incorporate perceptive examination, computational etymology, content mining, data extraction. A review made by the client concerning unmistakable substances, occasions or articles, and its features is alluded as realities. Additionally, an expression that portrays the person's Sentiment, assessments and the presentation examination with respect to elements, items, occasions and its characteristics is alluded as sentiment. Regularly Sentiments can be passed on everything that might be an individual, item, specific theme or business enterprises. This determines, the most opinions are passed on features that are having own components and attributes.

\section{Proposed Methodology - Convolutional Neural NeTWORK BASED QUick SENTIMENT ANALYSIS (CNN-QSA)}

Convolutional neural networks are the neural system that is utilized for handling information that has realized framework like topology. Natural language preparing task is utilizing convolutional neural systems, abuse the 1D structure of text information for accurate estimation. A convolutional neural system gives prevalent characterization precision as a result of the non-linearity of the system just as the capacity to effectively incorporate pre-prepared word embeddings. The text is classified from the sentiments using $\mathrm{CNN}$ is depicted in Figure 3.

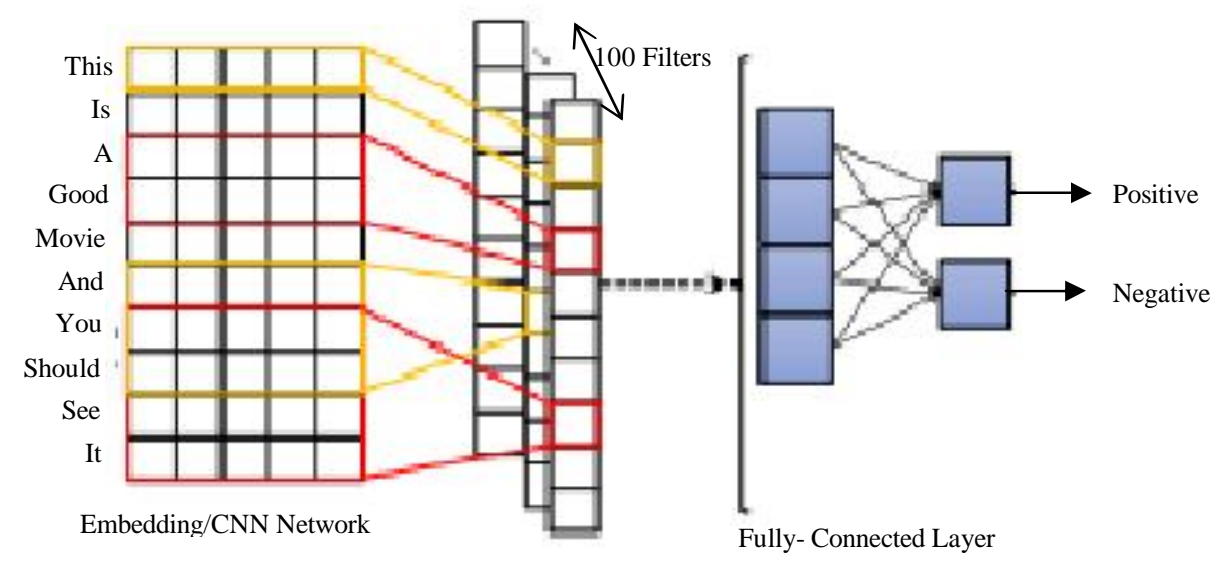

Figure 3: Text Classification using CNN

Convolutional neural systems are systems with convolutional and pooling layers, which are helpful for order undertakings, for example, assessment arrangement and so forth. At convolutional neural systems, convolutional and pooling designs are applied to text. Convolution is a specific sort of direct activity and pooling is an activity in convolutional neural systems. The convolution activity is done as 
Kota Venkateswara Rao et al., International Journal of Emerging Trends in Engineering Research, 8(9), September 2020, 5279- 5288

$$
\mathrm{C}(\mathrm{t})=(\mathrm{M} * \mathrm{w})(\mathrm{t})
$$

$$
\stackrel{d}{d W}=\max \left(d_{1}, d_{2}, \ldots, d_{n}\right)
$$

Here, * is the Convolutional operation $\mathrm{C}(\mathrm{t})$ - referred to as feature map, $\mathrm{M}$ is referred to as input $\mathrm{w}$ is referred to as the function such as kernel. The proposed Neural Network based Quick Sentiment Analysis (CNN-QSA) works as follows When a low dimensional vector is fed to the convolutional neural network to predict the sentiment is represented as.

$$
S(t)=d w_{1}=f(w x+T)
$$

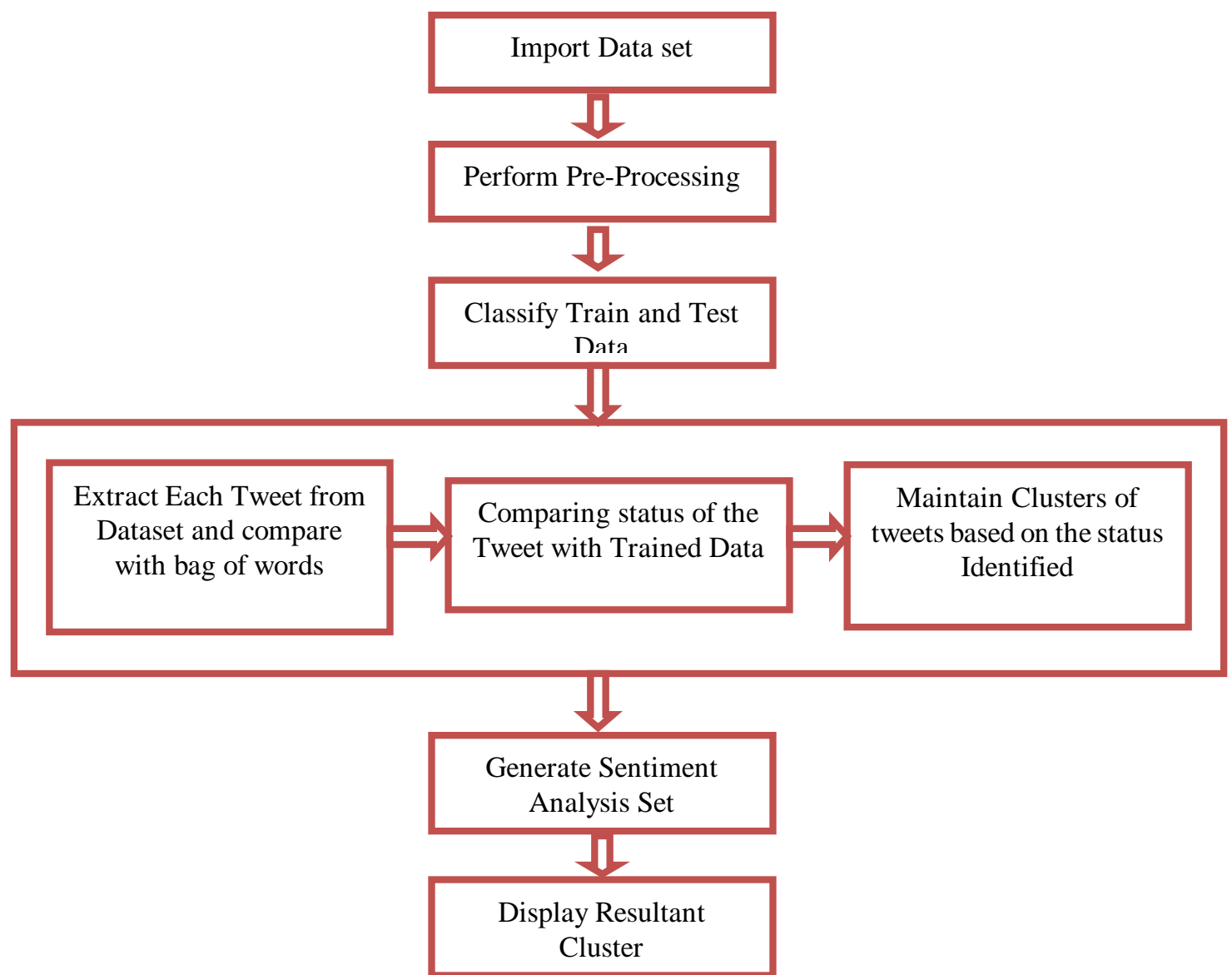

Figure 4: Over all Architecture of Proposed CNN-QSA

For Text classification, the input document or sentences was classified into two or three class classification. The output of the predicted sentiment of the document Di is represented as follows:

$$
D i=f(w x)=\sigma\left(W \cdot r_{v i}(x)+T\right)
$$

The proposed CNN-QSA model performs sentiment analysis on the given data using the algorithm given

In the proposed algorithm $\operatorname{Tr}(\mathrm{DS})$ is the Dataset considered having trained and test data that is given as input, $\mathrm{q}$ is the initial tweet considered from the dataset given. C(i) is the cluster set starting from initial record i.

Input: Dataset $\operatorname{Tr}(\mathrm{DS})$

Output: Given Tweets status is displayed in clusters

[1]. Initialize Bag of words, $i=1$
[2]. For each tweet q, do Apply Pre processing Eliminate Hashtags Eliminate Stop words end for Eliminate Special characters

[3]. Consider Selected token set TS

[4]. foreach $q € \operatorname{Tr}(\mathrm{DS})$ do

C(i) $\leftarrow$ q.tweet where q $\varepsilon \operatorname{Tr}(D S)$

Where $\mathrm{q}$ is the tweet considered from the given dataset $\operatorname{Tr}(\mathrm{DS})$ foreach $\mathrm{t} C(\mathrm{i}) \varepsilon \operatorname{Tr}(\mathrm{DS})$ do

$\mathrm{C}(\mathrm{i}+1)=\mathrm{q} \cdot \operatorname{sub}$ ("\#Bag of words) Where $\mathrm{C}(\mathrm{i}+1)$ is the sequential tweets considered. end for 
[5]. I = str.compare( C(i), Tr(Ds).Status. Positive if $(\mathrm{I}==0)$

C(i).Status =

q.sentiment('Positive'); end if

[6]. I = str.compare( C(i), Tr(Ds).Status. Negative if $(\mathrm{I} !=0)$

$\mathrm{C}(\mathrm{i}) \cdot$ Status $=$

q.sentiment('Negative');

else

C(i).Status =

q.sentiment('Neutral'); end if

[7]. Display Tweets status cluster sets $\mathrm{C}(\mathrm{i}) \varepsilon \operatorname{Tr}(\mathrm{DS})$ that represents positive, negative and neutral tweets.

\section{MATHEMATICAL MODELING OF CNN-QSA}

The proposed CNN-QSA have fast learning speed and improved execution of speculation. It can't to adjust the underlying parameters of concealed layer about each nonlinear undertakings are expressed as covered neurons. Thus, $\mathrm{N}$ discretionary particular examples $\{(\mathrm{pi}, \mathrm{qi}) \mid \mathrm{pi} \in \mathrm{R} \mathrm{n}$, qi $\in \mathrm{R} \mathrm{m}, \mathrm{i}=1, \ldots, \mathrm{N}\}$ in the resultant work with $\mathrm{L}$ covered neurons is given as

$\mathrm{f}_{\mathrm{L}}(\mathrm{p})=\sum_{\mathrm{i}=1}^{\mathrm{L}} \mathrm{w}_{\mathrm{i}} \mathrm{hl}_{\mathrm{j}}(\mathrm{p})=\mathrm{hl}(\mathrm{p}) \omega$

in which, $\omega=[\omega 1, \omega 2, \ldots$.$] : indicates the result vector$ among the $\mathrm{L}$ neurons and the resultant neuron of concealed layer, $\mathrm{hl}(\mathrm{x})=[\mathrm{hl}(\mathrm{p}), \mathrm{hl} 2(\mathrm{p}), \ldots \mathrm{hlL}(\mathrm{p})]$ : shows the resultant vector of information as of information space. So as to limit the preparation inaccuracy and to upgrade the presentation of speculation in neural system, it is conceivable to limit the preparation inaccuracy and the loads in a similar time and it is given by

$$
\operatorname{minimum}\left(\left\|H_{\omega}-\mathrm{T}\right\|,\|\omega\|\right)
$$

Right now, set of $\mathrm{s}$ channels is applied to a sliding window of length $\mathrm{h}$ over each sentence. Let $\mathrm{S}[\mathrm{i}: \mathrm{i}+\mathrm{h}]$ mean the connection of word vectors si to $\mathrm{si}+\mathrm{h}$. A component CIi is produced for a given channel $\mathrm{R}$ by:

$$
\mathrm{CI}_{\mathrm{j}}:=\sum_{\mathrm{k}_{\mathrm{i} j}}\left(\mathrm{~S}_{[\mathrm{i} i \mathrm{i}+\mathrm{h}]}\right)_{\mathrm{k}, \mathrm{j}} \mathrm{R}_{\mathrm{k}, \mathrm{y}}
$$

There is a filter which parameterized by the weight matrix WM with region size $S$; the sentence matrix $A \in \mathbb{R}^{B \times d}, A[i ; j]$ is the sub-matrix of $K$ from row $i$ to row $j$. The output $o \in \mathbb{R}^{z-h+1}$ can be calculated
$\mathrm{K}=\pi \mathrm{r}^{2} \mathrm{o}_{\mathrm{i}}=$ WM.A[i: $\left.\mathrm{i}+5-1\right]$

In the formula $\mathrm{i}=1 \ldots \mathrm{s}-\mathrm{h}+1$ is the dot product between sub-matrix and the filter. Same as other neural network model, bias $b \in \mathbb{R}$ and activation function $\mathrm{f}$ have been added to $o_{\mathrm{i}}$ and the feature map $\mathrm{c} \in \mathbb{R}^{\mathrm{z}-\mathrm{h}+1}$ for

$c_{\mathrm{i}}=f\left(o_{\mathrm{i}}+\mathrm{b}\right)$

The basic $n$-gram language model is used to assign a probability $\mathrm{P}(\mathrm{t})$ to every possible word sequence $\mathrm{W}$ i.e. the feature derived are represented as:

$P(t)=\sum_{i=1}^{v}\left(w_{i} \mid w_{i-1}\right)+c i+K$

WBOW $\left(\mathrm{f}_{2}, \mathrm{f}_{2}, \ldots, \mathrm{f}_{\mathrm{k}}\right)=\frac{1}{\sum_{\mathrm{i}}^{\mathrm{k}} \mathrm{WV}_{\mathrm{i}}} \sum_{\mathrm{i}}^{\mathrm{k}} \mathrm{v}\left(\mathrm{f}_{\mathrm{i}}\right)-\mathrm{P}(\mathrm{t})+\mathrm{k}$

(11)

Consequently $\mathrm{O}(\mathrm{p})$ is the output function and is represented as

$O(p)=\left[k\left(p, p_{1}\right), \ldots k\left(p, p_{N}\right)\right](M+k)^{-1}$

On the off chance that attribute Ai with values $\{\mathrm{v} 1, \mathrm{v} 2, \ldots . . \mathrm{vN}\}$ is utilized for tree root, at that point it sub separates $\mathrm{P}$ into $\{\mathrm{P} 1, \mathrm{P} 2, \ldots \ldots \mathrm{PN}\}$, in which Pi determines the classes in $\mathrm{T}$ that have esteem vi of Xi. Expect Ti involves ai classes of $\mathrm{O}(\mathrm{p})$; bi classes of WBOW and ci classes of $\mathrm{P}(\mathrm{t})$, at that point the mediator data which is required for the sub-tree for Ti are $\mathrm{I}(\mathrm{ai}$ ,bi), I(bi ,ci) and I(ai ,ci). The $\mathrm{L}$ anticipated data required for the decison tree with $\mathrm{Ri}$ as the $(\mathrm{L}(\mathrm{Xi}))$, is set up as a weighted normal are given as follows:

$L\left(X_{1}\right)=\sum_{i=1}^{N} \frac{a_{i}+b_{i}}{a+b} I\left(a_{i}, b_{i}\right)$

\section{RESUlTs \& DisCUSSIONS}

The proposed method for performing sentiment analysis is done using ANACONDA SPYDER and dataset that is utilized for assessing the evaluation is available at http://www.cs.cornell.edu/individuals/pabo/film audit information and movie review dataset used is the recently proposed Stanford Sentiment Treebank (SSTb). The dataset organizer have „txt_sentoken ${ }^{\text {ee }}$ which are the 2000 processed down-cased content documents that are used in Pang/Lee $\mathrm{ACL}$ and there are two subdirectories that are "pos" and "nege, it determines the genuine review of the segment records dependent on the proposed programmed rating classifier and results are clearly depicted. The original training set contains 1.5 million tweets that were automatically labeled as positive/negative using emoticons as 
Kota Venkateswara Rao et al., International Journal of Emerging Trends in Engineering Research, 8(9), September 2020, $5279-5288$

noisy labels. The datasets considered and the tweets considered for training and testing are represented in Table 1.

Table 1: Trained and Test Tweets

\begin{tabular}{|c|c|c|c|}
\hline Dataset & Set & Tweets & classes \\
\hline \multirow{2}{*}{$\begin{array}{c}\text { Film Audit } \\
\text { Dataset }\end{array}$} & $\begin{array}{l}\text { Trai } \\
\mathrm{n}\end{array}$ & 96521 & 5 \\
\cline { 2 - 4 } & Test & 2541 & 5 \\
\hline \multirow{2}{*}{ SSTb } & $\begin{array}{l}\text { Trai } \\
\mathrm{n}\end{array}$ & 85247 & 3 \\
\cline { 2 - 4 } & Test & 1556 & 3 \\
\hline
\end{tabular}

An assessment metric is used to quantify the value of sentiment analysis frameworks and to clarify the theoretical and useful advancements of these frameworks. It contains measurements which trails the general unrevealed procedure of assessment. Not many of the measures that are chosen with the end goal of assessment are Recall, Precision, and the F-Measure. The performance of the proposed classifier is contrasted with the traditional methods and are depicted in Table 2.

Table 2: Performance levels of Various Classifiers

\begin{tabular}{|c|c|c|c|}
\hline $\begin{array}{c}\text { Classifier } \\
\text { Model }\end{array}$ & Precision & Recall & F-Measure \\
\hline SVM & 0.56 & 0.62 & 0.72 \\
\hline LSTM & 0.68 & 0.57 & 0.76 \\
\hline CART & 0.85 & 0.72 & 0.82 \\
\hline $\begin{array}{c}\text { CNN-QS } \\
\text { A }\end{array}$ & 0.97 & 0.95 & 0.93 \\
\hline
\end{tabular}

The predicted values are depicted in Table 3 . The Trained and Test dataset parameters data is clearly depicted specifying positive, negative and neutral tweets.

Table 3: Levels of Predicted Values

\begin{tabular}{|c|c|c|c|}
\hline $\begin{array}{c}\text { Predicte } \\
\text { d Value }\end{array}$ & $\begin{array}{c}\text { Positive } \\
\text { Category }\end{array}$ & $\begin{array}{c}\text { Negative } \\
\text { Categor } \\
\mathbf{y}\end{array}$ & $\begin{array}{c}\text { Neutral } \\
\text { Categor } \\
\mathbf{y}\end{array}$ \\
\hline $\begin{array}{c}\text { Trained } \\
\text { dataset }\end{array}$ & 21365 & 12352 & 568 \\
\hline $\begin{array}{c}\text { Test } \\
\text { dataset }\end{array}$ & 8652 & 3657 & 103 \\
\hline
\end{tabular}

For better use of this proposed technique for the proficient characterization of reviews with mining, it needs the estimations of these measures to be determined. The estimations of measures are resolved based on True Positive (TP), True Negative (TN), False Positive (FP) and False Negative (FN) with the decision of customer review classification. The examination of ROC is utilized to figure the classifier accuracy. The flat and vertical pivot of ROC curve is

given as $x=1-\operatorname{specificity}(t)$ and $y=\operatorname{sensitivity}(t)$.

Figure 5 outlines the partition with the cut-off point for best explicitness and affectability. The proposed model to the preparation information and produces the client survey information. It is discovered that the pace of affectability of estimation examination for the proposed approach is about $93 \%$ and explicitness pace of $90 \%$. The ROC curves levesl of the proposed method is compared with the traditional LSTM (longshort-term-memory) and SVM method.

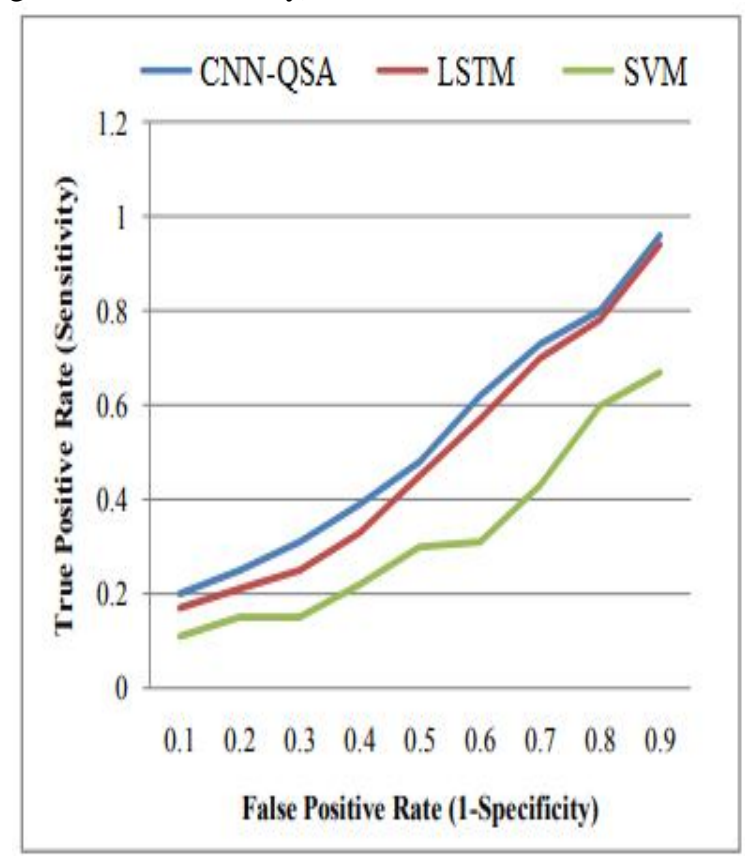

Figure 5: ROC Curve Levels

The precision evaluates the number of reviews that are to be categorized as Positive (Negative or Neutral) is actually Positive (Negative or Neutral) using the equation:

precision $=\frac{\text { True Positive }}{\text { False Positive }+ \text { True Positive }}$

The recall specifies the numbers of reviews of Positives (Negatives or Neutrals) classes actually are categorized. The accurate classification percentage of Positives (Negatives or Neutrals) is specified using recall. It is also identical to Sensitivity:

Recall $=\frac{\text { True Positive }}{\text { False Negative }+ \text { True Positive }}$

F-Measure integrates the precision and recall and it is considered as the harmonic mean of precision and recall:

F- Measure $-\frac{2 \text { (Precision } \times \text { Recall) }}{\text { precision }+ \text { Recall }_{1}}$ 
Accuracy evaluates the weighted percentage of reviews Positive, Negative and Neutral which are categorized accurately.

$$
\text { Accuracy }=\frac{T P+T N}{T P+F P+T N+F N} \times 100
$$

The time levels of the proposed method is depicted in Table 4. The proposed method takes less time in analysis of the sentiment tweets.

Table 4: Time Level Parameters

\begin{tabular}{|c|c|c|c|}
\hline $\begin{array}{c}\text { Classifie } \\
\text { r Model }\end{array}$ & $\begin{array}{c}\text { Produc } \\
\text { t Tweets } \\
\text { Count }\end{array}$ & $\begin{array}{c}\text { Trainin } \\
\text { g Time } \\
\text { levels } \\
\text { (hrs.min) }\end{array}$ & $\begin{array}{c}\text { Testing } \\
\text { Time levels(in } \\
\text { milliseconds) }\end{array}$ \\
\hline SVM & 36524 & 4.37 & 19 \\
\hline LSTM & 38981 & 3.23 & 16 \\
\hline $\begin{array}{c}\text { CNN-QS } \\
\text { A }\end{array}$ & 45867 & 1.09 & 8 \\
\hline
\end{tabular}

The processing time of the proposed CNN-QSA method is very low when compared to traditional methods. Figure 6 represents the processing time levels.

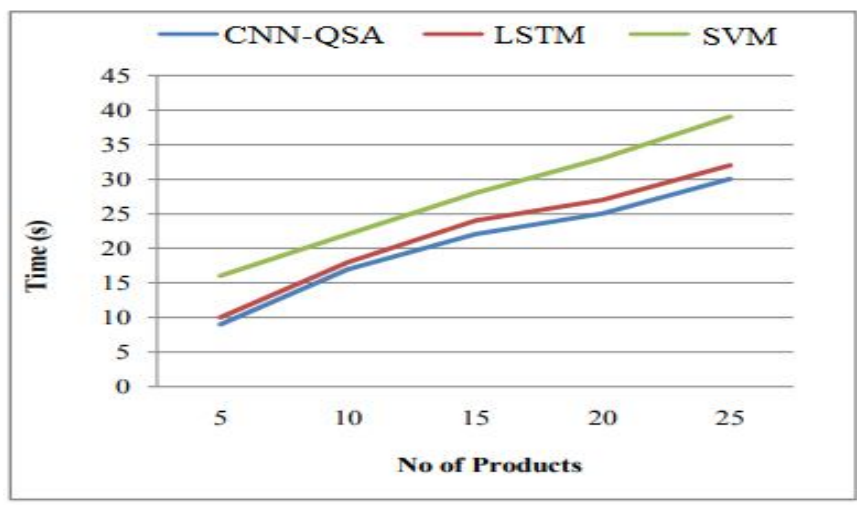

Figure 6: Time levels for Processing Sentiments

The accuracy, sensitivity and specificity of the proposed method and traditional methods parameters are depicted in Table 5.

Table 5: Performance Parameters

\begin{tabular}{|c|c|c|c|}
\hline $\begin{array}{c}\text { Classifier } \\
\text { Model }\end{array}$ & Accuracy & Sensitivity & Specificity \\
\hline SVM & 87 & 83 & 80 \\
\hline LSTM & 93 & 91 & 86 \\
\hline CNN-QSA & 96 & 94 & 90 \\
\hline
\end{tabular}

The comparison of accuracy, sensitivity and specificity of the proposed method is compared with the traditional methods and the results depict that the proposed method exhibits better performance. Figure 7 represents the comparison levels.

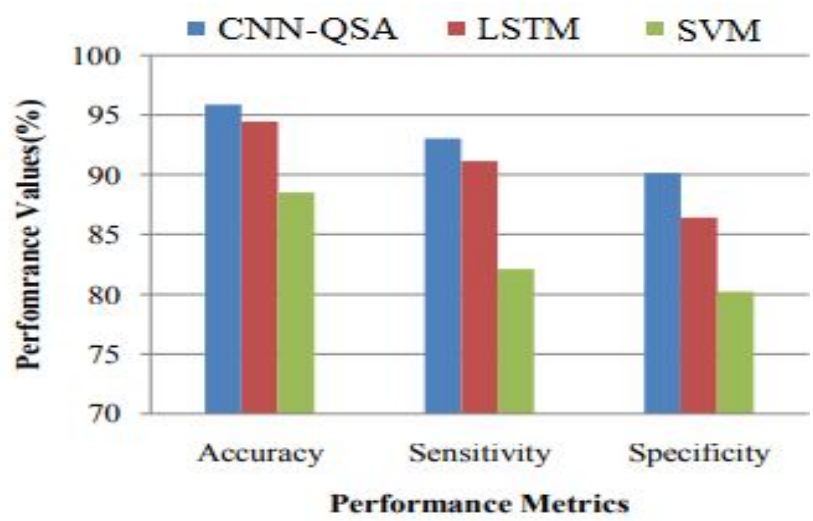

Figure 7: Comparison Levels

The comparison of precision, recall and F-measure of the proposed method is compared with the traditional methods and the results depict that the proposed method exhibits better performance. Figure 8 represents the comparison levels.

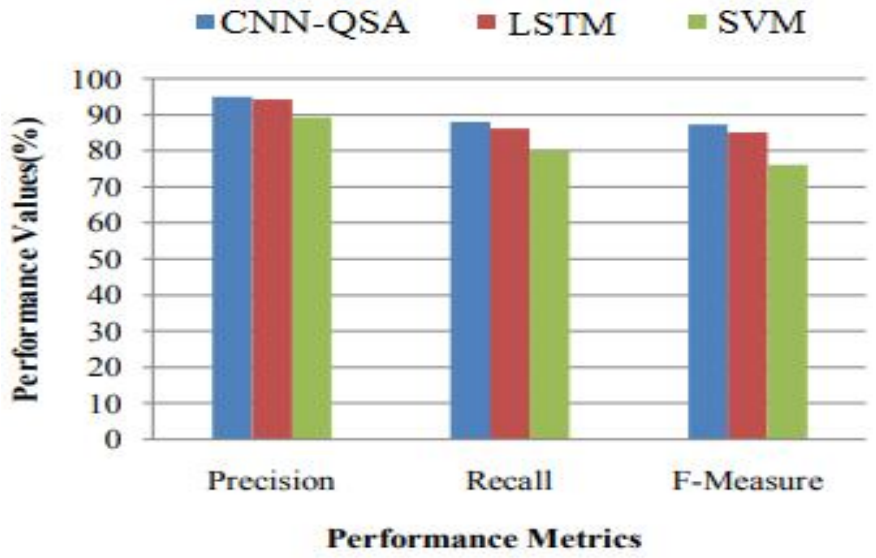

Figure 8: Comparison Levels of the Parameters

The training accuracy levels of the proposed method with positive, negative and neutral words are depicted in Figure 9.

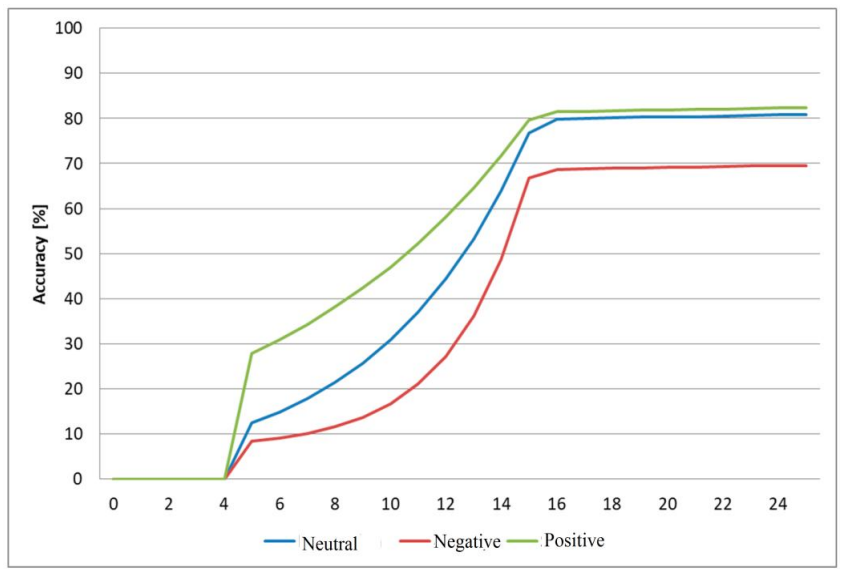

Figure 9: Training Levels

The testing accuracy levels of the proposed method with positive, negative and neutral words are depicted in Figure 10. 


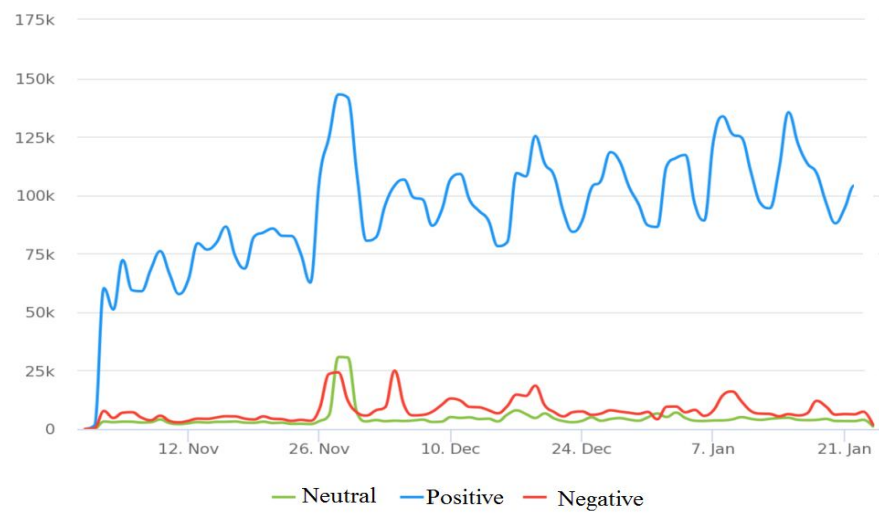

Figure 10: Testing Levels

The proposed CNN-QSA algorithm is compared with the existing LSTM and SVM methods and the accuracy of the models are depicted in Table 6.

Table 6: Accuracy Levels

\begin{tabular}{|l|l|}
\hline Algorithm & Accuracy \\
\hline CNN-QSA & $93 \%$ \\
\hline LSTM & $84 \%$ \\
\hline SVM & $76 \%$ \\
\hline
\end{tabular}

\section{CONCLUSION}

Sentiment Analysis using machine learning methods is ordinarily utilized in opinion mining for knowing notions, subjectivities also delicate states in online reviews and opinions. The procedure was practiced on product assessment by sorting out the items qualities. A convolutional neural network model joins the qualities of keen words to manage the issue of Sentiment analysis. A keen word lexicon coordinating the features of the useful words into the word vector when preparing the word vectors, finally a convolutional neural system strategy was familiar with performing Sentiment analysis. The word vector joins the qualities of adoring words was input, and the result was the extremity of the remark data. Contrast with the conventional strategy, this technique improves execution. Be that as it may, there are still spaces for development, the measure of preparing information is little which may cause model preparing be inadequate This methodology hence can be considered to join information on the morphological structure of sentences, with the inducing intensity of CNNs. In future work, investigating the impact of different mixes of part-of speech labels and other linguistic structures, on expectation accuracy should be possible.

\section{REFERENCES}

1. Y. Han and K. K. Kim. Sentiment analysis on social media using morphological sentence pattern model, IEEE 15th International Conference on Software
Engineering Research, Management and Applications (SERA), London, 2017, pp. 79-84, 2017.

2. Z. Nasim, Q. Rajput and S. Haider. Sentiment analysis of student feedback using machine learning and lexicon based approaches, International Conference on Research and Innovation in Information Systems (ICRIIS), Langkawi, 2017, pp. 1-6, 2017.

3. Z. Xiangyu, L. Hong and W. Lihong. A context-based regularization method for short-text sentiment analysis, International Conference on Service Systems and Service Management, Dalian, 2017, pp. 1-6, 2017.

4. M. H. Krishna, K. Rahamathulla and A. Akbar, A feature based approach for sentiment analysis using SVM and coreference resolution, International Conference on Inventive Communication and Computational Technologies (ICICCT), Coimbatore, pp. 397- 399, 2017.

5. P. Yadav and D. Pandya. SentiReview: Sentiment analysis based on text and emoticons, International Conference on Innovative Mechanisms for Industry Applications (ICIMIA), Bangalore, pp. 467-472. 2017.

6. M. Trupthi, S. Pabboju and G. Narasimha. Sentiment Analysis on Twitter Using Streaming API," IEEE 7th International Advance Computing Conference (IACC), Hyderabad, 2017, pp. 915-919, 2017.

7. K. Liu, Y. Niu, J. Yang, J. Wang and D. Zhang. Product Related Information Sentiment-Content Analysis Based on Convolutional Neural Networks for the Chinese Micro-Blog, International Conference on Network and Information Systems for Computers (ICNISC), Wuhan, 2016, pp. 357-361, 2016.

8. D. V. N. Devi, C. K. Kumar and S. Prasad. A Feature Based Approach for Sentiment Analysis by Using Support Vector Machine, IEEE 6th International Conference on Advanced Computing (IACC), Bhimavaram, pp. 3-8, 2016.

9. R. Hegde and Seema S.Aspect based feature extraction and sentiment classification of review data sets using Incremental machine learning algorithm. Third International Conference on Advances in Electrical, Electronics, Information, Communication and BioInformatics (AEEICB), Chennai, 2017, pp. 122-125, 2016.

10. K. Han, K. Youngsub, Y. Kim, and Jin-Hee Song. Building Sentiment Lexicon for Social Media Analysis using Morphological Sentence Pattern Model, Advanced Science and Technology Letters, 136, pp. 103-106, 2016.

11. S. Jebbara and P. Cimiano. Aspect-Based Sentiment Analysis Using a Two-Step Neural Network Architecture," Communications in Computer and Information Science, vol 641, 2016.

12. Ghiassi, M.; Lee, S. A domain transferable lexicon set for Twitter sentiment analysis using a supervised machine learning approach. Expert Syst. Appl. 106, 197-216, 2018. 
13. Balazs, J.A.; Velásquez, J.D. Opinion mining and information fusion: A survey, Inf. Fusio, , 27, 95-110, 2017.

14. Chaturvedi, I.; Cambria, E.; Welsch, R.E.; Herrera, F. Distinguishing between facts and opinions for sentiment analysis: Survey and challenges. Inf. Fusio, 44, 65-77, 2018.

15. C.R.Bharathi, Vejendla. Lakshman Narayana, L.V. Ramesh," Secure Data Communication Using Internet of Things, International Journal of Scientific \& Technology Research, 9(4), pp:3516-3520, 2020.

16. Evgeniy Ivanovich Trubilin, Svetlana Ivanovna Borisova, Vladimir Ivanovich Konovalov, Mikhail. Experimental Studies of Parameters of Pneumatic Slot Sprayer, International Journal of Emerging Trends in Engineering Research, 8(1), 2020.

17. M. Shyamala Devi, Shefali Dewangan, Satwat Kumar Ambashta, Anjali Jaiswal, Nariboyena Vijaya Sai Ram, Backward Eliminated Formulation of Fire Area Coverage using Machine Learning Regression, International Journal of Innovative Technology and Exploring Engineering, 8(12), pp.1565-1569, 2019.

18. M. Shyamala Devi, Ankita Shil, Prakhar Katyayan, Tanmay Surana. Constituent Depletion and Divination of Hypothyroid Prevalance using Machine Learning Classification, International Journal of Innovative Technology and Exploring Engineering, 8(12), pp. 1607-1612, 2019.

19. Hennadii Khudov, Irina Khizhnyak, Fedor Zots, Galina Misiyuk, Oleksii Serdiu. The Bayes Rule of Decision Making in Joint Optimization of Search and Detection of Objects in Technical Systems, International Journal of Emerging Trends in Engineering Research, 8(1), 2020.

20. JKR Sastry, M Trinath Basu, Multi-Factor Authentication through Integration with IMS System, International Journal of Emerging Trends in Engineering Research, 8(1), 2020.

21. M. Shyamala Devi, Shefali Dewangan, Satwat Kumar Ambashta, Anjali Jaiswal, Sairam Kondapalli. Recognition of Forest Fire Spruce Type Tagging using Machine Learning Classification, International Journal of Recent Technology and Engineering, 8 (3), pp. 4309 4313, 2019.

22. M. Shyamala Devi, Usha Vudatha, Sukriti Mukherjee, Bhavya Reddy Donthiri, S B Adhiyan, Nallareddy Jishnu, Linear Attribute Projection and Performance Assessment for Signifying the Absenteeism at Work using Machine Learning, International Journal of Recent Technology and Engineering, 8 (3), pp. 1262 1267, 2019. 